\section{Can we prevent}

\section{Alzheimer's disease?}

\author{
Podemos prevenir
}

\section{a doença de Alzheimer?}

Dementia is one of the most challenging health problems of the XXI Century. Current estimates suggest that by 2030 there will be 63 million people with dementia worldwide, but our societies remain largely unprepared to deal with the devastating personal, social and financial impact of such an epidemic. Currently available therapies for Alzheimer's disease (AD), which is the most frequent cause of dementia, are unable to change the course of the illness and have questionable costeffectiveness. Various groups around the world are now actively engaged in the search for an effective treatment for $A D$, as well as the development of interventions that may prevent (or delay) the clinical manifestation of the illness. The approaches being tested are based on our partial understanding of the mechanisms that ultimately lead to the development of $A D$; the most obvious example being the $ß$-amyloid vaccine.

B-amyloid is a peptide found in senile plaques, which is one of the key neuropathological abnormalities of AD. The introduction of the B-amyloid transgenic mice enabled scientists to test the effects of an anti-amyloid vaccine. They found that vaccinated mice were able to clear B-amyloid from their brains (the same was later observed in humans). A phase Ila trial was then started to test the effect of the vaccine in people with $A D$, but had to be discontinued a few months later due to the development of severe encephalitis and death amongst study participants. ${ }^{1}$ Disappointingly, the vaccinated subjects who survived experienced no obvious clinical benefit from treatment. These results challenge the very core of the amyloid cascade hypothesis of AD and suggest that the B-amyloid peptide may not be its most critical pathological abnormality.

Other approaches to treat and prevent AD are currently being considered. These are mostly based on the findings of observational studies examining the association between cardiovascular risk factors and dementia, such as the one recently published by Richard Mayeux's group. ${ }^{2}$ They followed a cohort of 1138 older people free of dementia for a mean period of 5.5 years. They investigated the risk of AD associated with four known cardiovascular risk factors: diabetes, hypertension, heart disease and smoking. The results of the study showed that the risk of $A D$ increased with the number of cardiovascular risk factors: hazard ratio of $1.7(95 \%$ confidence interval; $95 \% \mathrm{Cl}: 1.1,2.4), 2.6(95 \% \mathrm{Cl}: 1.6,3.9)$ and $3.4(95 \% \mathrm{Cl}: 2.1,5.7)$ for one, two and three or more risk factors. If there is a causal association between cardiovascular risk factors and $A D$, then the management of such factors should reduce the incidence of $A D$.

Five randomised, placebo-controlled trials of hypertension have investigated cognitive function and dementia as an outcome of interest. The MRC and SHEP trials, which used diuretics and B-blockers, found no obvious cognitive benefit associated with treatment. The SYST-EUR and PROGRESS used the ACE-inhibitors enalapril and perindopril (with or without hydrochlorithiazide or indapamide). The median followup of patients in the SYST-EUR was 2 years. The incidence of dementia was reduced from 7.7 in the placebo group to $3.7 / 1000$ patient-years in the active treatment group. Although the difference between the study groups was statistically 
significant, the findings were based on only 32 incident cases of vascular or mixed dementia, or AD. The PROGRESS recruited subjects with a positive history of stroke and a wide range of blood pressure at entry. Treatment with perindopril was associated with a non-significant reduction of $12 \%$ $(95 \% \mathrm{Cl} ;-8 \%, 28 \%)$ in the risk of dementia after 4 years. The last and largest trial to date was the SCOPE. ${ }^{3}$ The study randomised 4964 hypertensive patients aged 70 to 89 years to treatment with candesartan, and ACE-inhibitor, or placebo. Participants were followed for 3.7 years on average. Significant cognitive decline was noted in 13.5 and 15.2/1000 personyears treated with candesartan and placebo, whereas dementia was diagnosed in 6.8 and $6.3 / 1000$ person-years respectively $(p>0.20)$. Taken together, these results suggest that antihypertensive treatment is unlikely to be an effective strategy to prevent cognitive decline and dementia in later life.

The management of other cardiovascular risk factors, such as diabetes and high lipids, has so far produced equivocal results in relation to cognitive outcomes, in spite of the promising associations reported by observational studies. Likewise, the purported neuroprotective effects of estradiol failed to materialise in the Women Health Initiative trial. ${ }^{4}$ Worryingly, the results of that study showed that women treated with hormone replacement had almost twice the risk of cognitive impairment and dementia when compared to controls. . $^{-5}$ There is also limited evidence from randomised trials that the use of folate and vitamin B12 to reduce total plasma homocysteine decreases the risk of dementia or cognitive decline (high plasma homocysteine has been associated with increased risk of strokes and $A D$ in observational studies). The management of other factors associated with dementia, such as physical or mental activity and use of antioxidants, has not as yet been rigorously tested by randomised trials. As a result, it is not possible to determine whether they represent a practical alternative to reduce dementia risk. If they do, their contribution to risk reduction is likely to be small.

And where does that leave us? The current treatment of AD has limited efficacy and poor cost-effectiveness. Interventions designed to prevent $A D$ by targeting known risk factors have produced questionable, negative or, in the case of estrogen and the B-amyloid vaccine, harmful results. It seems unlikely, at this stage, that our current knowledge about the pathogenesis of AD would be sufficient to guide the introduction of effective preventative strategies. Scientists and policy makers will have to work very hard over the next few years to find an acceptable solution to the enormous challenge created by the increasing number of people with dementia in our societies.

Osvaldo P Almeida

Unit of Geriatric Psychiatry, University of Western Australia

\section{Acknowledgements}

This work was partly supported by a project grant from the National Health and Medical Research Council of Australia.

Conflict of interests: None declared

\section{References}

1. Nicoll JA, Wilkinson D, Holmes C, Steart P, Markham H, Weller RO. Neuropathology of human Alzheimer disease after immunization with amyloid-beta peptide: a case report. Nat Med. 2003;9(4):448-52.

2. Luchsinger JA, Reitz C, Honig LS, Tang MX, Shea S, Mayeux R. Aggregation of vascular risk factors and risk of incident Alzheimer disease. Neurology. 2005;65(4):545-51.

3. Lithell H, Hansson L, Skoog I, Elmfeldt D, Hofman A, Olofsson B, et al. The Study on Cognition and Prognosis in the Elderly (SCOPE): principal results of a randomized double-blind intervention trial. J Hypertens. 2003;21(5):875-86.

4. Shumaker SA, Legault C, Kuller L, Rapp SR, Thal L, Lane DS, et al. Conjugated equine estrogens and incidence of probable dementia and mild cognitive impairment in postmenopausal women: Women's Health Initiative Memory Study. JAMA. 2004;291(24):2947-58.

5. Almeida OP, Flicker L. Association between hormone replacement therapy and dementia: Is it time to forget? Int Psychogeratr. 2005;17(2): 155-64. 